\title{
Effects of relativistic electron temperature on parametric instabilities for intense laser propagation in underdense plasma
}

\author{
Yao Zhao, ${ }^{1}$ Jun Zheng, ${ }^{1}$ Min Chen, ${ }^{1}$ Lu-Le Yu, ${ }^{1}$ Su-Ming Weng, ${ }^{1}$ Chuang Ren, ${ }^{2,3}$ Chuan-Sheng Liu, ${ }^{4}$ and \\ Zheng-Ming Sheng ${ }^{1,5, \text { a) }}$ \\ ${ }^{1)}$ Key Laboratory for Laser Plasmas (MoE) and Department of Physics and Astronomy, Shanghai Jiao Tong University, \\ Shanghai 200240, China \\ ${ }^{2)}$ Department of Mechanical Engineering and Laboratory for Laser Energetics, University of Rochester, Rochester, \\ New York 14627, USA \\ ${ }^{3)}$ Department of Physics and Astronomy, University of Rochester, Rochester, New York 14627, \\ USA \\ ${ }^{4)}$ East-West Space Science Center, University of Maryland, College Park, Maryland 20742, \\ USA \\ ${ }^{5}$ SUPA, Department of Physics, University of Strathclyde, Glasgow G4 ONG, UK \\ (Dated: 8 November 2014)
}

Effects of relativistic electron temperature on stimulated Raman scattering and stimulated Brillouin scattering instabilities for high intensity lasers propagating in underdense plasma are studied theoretically and numerically. The dispersion relations for these instabilities are derived from the relativistic fluid equation. For a wide range of laser intensity and electron temperature, it is found that the maximum growth rate and the instability region in $k$-space can be reduced at relativistic electron temperature. Particle-in-cell simulations are carried out, which confirm the theoretical analysis.

\section{INTRODUCTION}

The propagation of short intense lasers in underdense plasma has been intensively studied, since it is closely related to a number of applications such as laser-driven particle acceleration $^{1}$, advanced concept of inertial confined fusion including fast ignition with electron or ion beams ${ }^{2-4}$, shock ignition $^{5}$, impact ignition ${ }^{6}$, and so on. Earlier studies ${ }^{7}$ on short-pulse laser plasma interactions are mostly based on the cold plasma model assuming that the plasma electrons cannot be heated to high temperature by short pulse lasers in a short interval of time. However, theory and simulations suggest that relativistic high temperatures at the multi-MeV level can be achieved by intense pulses quickly. ${ }^{8-12}$ Therefore, the trailing parts of laser pulses often interact with plasmas with high temperatures. Moreover, in the case of multi-pulse laser plasma interaction, the following-up pulses may encounter high temperature plasma created by foregoing laser pulses. It is therefore interesting to consider the effects of relativistic plasma temperatures on laser propagation.

Several theoretical and numerical studies have been reported on the interaction of laser with relativistic hot plasmas. ${ }^{13-15}$ Tzeng et al. ${ }^{13}$ have shown that the plasma temperature can suppress the relativistic self-focusing of laser; Bergman and Eliasson ${ }^{14}$ have studied the plasma dispersion relation in high temperature plasma; Li et al. ${ }^{15}$ have investigated the effect of plasma temperature on laser hosing instability. In these cases, the thermal velocity is comparable to the quiver velocity of the electrons in the laser fields and the electron mass is modulated by its thermal velocity too. The instability growth rate of stimulated Raman scattering (SRS) has been calculated analytically in relativistic hot plasma with Maxwellian ${ }^{16}$

a)zmsheng@sjtu.edu.cn or zhengming.sheng@strath.ac.uk or waterbag ${ }^{17,18}$ electron distribution function. These studies indicated that the hot plasma electron temperature tends to weaken the SRS instability. However, the Jüttner-Synge distribution is more suitable to describe the relativistic plasma, which is considerably different from either the Maxwellian distribution or the waterbag distribution to describe the laser propagation when plasma electron temperature $T_{e}$ is above $511 \mathrm{keV} .{ }^{19}$ Furthermore, the effects of relativistic temperature on simulated Brillouin scattering (SBS) are still not considered so far.

In this paper, we investigate the effects of relativistic plasma electron temperature on SRS and SBS instabilities by theoretical analyses and numerical simulations. In Sec. II, the dispersion relations for SRS and SBS are derived for plasmas with relativistic hot electrons. The growth rates are obtained numerically and the maximum growth rate of SRS and SBS are given under certain conditions. In Sec. III, particle-in-cell (PIC) simulations are carried out to investigate the development of SRS and SBS. Comparison is made between the cases of non-relativistic and relativistic plasmas. We summarize our results in section IV.

\section{ANALYTICAL MODELS FOR THE PARAMETRIC INSTABILITIES IN HOT PLASMA}

\section{A. Dispersion relation of SRS}

We start with the relativistic fluid equation of motion for electron $^{19,20}$,

$$
m_{e} \frac{\partial\left(\alpha \gamma \mathbf{v}_{e}\right)}{\partial t}=\frac{e}{c} \frac{\partial \mathbf{A}}{\partial t}+e \nabla \phi-\alpha m_{e} c^{2} \nabla \gamma-\frac{\nabla \mathcal{P}}{n_{e}}
$$

where $\mathbf{v}_{e}, e, n_{e}$ and $m_{e}$ are the electron fluid velocity, electron charge, electron density and electron mass, respectively. $\mathbf{A}$ is the vector potential and $\phi$ is the electrostatic poten- 
tial. $\gamma=1 / \sqrt{1-\mathbf{v}_{e} \cdot \mathbf{v}_{e} / c^{2}}$ is the relativistic factor. The dimensionless parameter $\alpha$ represents the relativistic mass increase with random motion of the particles. It is defined as $\alpha=(\mathcal{P}+\mathcal{E}) / \bar{n} m_{e} c^{2}$, where $\mathcal{P}$ is the isotropic pressure, $\mathcal{E}=m_{e}^{-2} c^{2} \int \gamma f(p) d^{3} p$ is the energy density, and $\bar{n}=n_{e} / \gamma$ is the particle density in the local rest frame. ${ }^{15}$ It is clear that $\alpha$ is a function of electron thermal velocity $\theta_{t e}=\sqrt{T_{e 0} / m_{e}}$. For cold plasmas, $\mathcal{P}=0, \mathcal{E}=\bar{n} m_{e} c^{2}$, hence $\alpha=1$. In our derivation, we assume that $\alpha$ is uniform in space. Here we wish to point out that the factor $\alpha$ depends significantly on the distribution function. In particular, the Jüttner-Synge distribution with $f(p)=\bar{n} \mu\left[4 \pi c K_{2}(\mu)\right]^{-1} \exp \left(-\mu \sqrt{1+p^{2} / m_{e}^{2} c^{2}}\right)$ where $\mu=m_{e} c^{2} / T_{e 0}$ and $K_{2}$ is the modified Bessel function of the second kind, gives a much larger $\alpha$ than the Maxwellian distribution for a given thermal velocity $\theta_{t e}$ when $\theta_{t e} / c>1 .^{15}$

To derive the dispersion relation for the SRS instability, the ions can be treated as a stationary and neutralizing background. Let us take the normalized laser amplitude $\mathbf{a}=$ $e \mathbf{A} / m_{e} c^{2}$, and use $\mathbf{v}_{e} \sim \mathbf{a} c / \alpha \gamma$, we have $\gamma=\sqrt{1+\mathbf{a}^{2} / \alpha^{2}}$ as shown in Ref. [15]. This means that the mass of relativistic hot particles increase with relativistic temperature and becomes heavy enough to be driven by laser, i.e., the relativistic temperature modulation suppresses the effects of laser. Writing $\mathbf{a}=\mathbf{a}_{0}+\widetilde{\mathbf{a}}$, where $\widetilde{\mathbf{a}}$ is a small perturbation, we can simplify $\gamma \simeq \gamma_{0}+\left(\mathbf{a}_{0} \cdot \widetilde{\mathbf{a}}\right) / \alpha^{2} \gamma_{0}$, where $\gamma_{0}=\sqrt{1+\mathbf{a}_{0}^{2} / \alpha^{2}}$. The SRS is considered as an adiabatic process with $p / n^{\beta}=$ const, where $\beta=(2+N) / N$ and $N$ is the number of freedom degrees. ${ }^{19}$ Using first order linearization, we write $\mathbf{v}_{e}=\mathbf{v}_{e 0}+\mathbf{v}_{e 1}, n_{e}=n_{e 0}+n_{e 1}, \phi=\phi_{1}$. Substituting these relations into Eq. (1) and using Poisson's equation along with the equation of continuity, one obtains the equation for the electron density fluctuation

$$
\left(\alpha \frac{\partial^{2}}{\partial t^{2}}+\omega_{p}^{\prime 2}-\frac{\beta \theta_{t e}^{2}}{\gamma_{0}} \nabla^{2}\right) \delta n_{e}=\frac{c^{2}}{\alpha \gamma_{0}^{2}} \nabla^{2}\left(\mathbf{a}_{0} \cdot \widetilde{\mathbf{a}}\right),
$$

where $\delta n_{e}=n_{e 1} / n_{e 0}, \omega_{p}^{\prime}=\sqrt{4 \pi n_{e 0} e^{2} / m_{e} \gamma_{0}}=\omega_{p} / \sqrt{\gamma_{0}}$.

With the usage of the Coulomb gauge $\nabla \cdot \mathbf{A}=0$, Ampere's law yields

$$
\left(\nabla^{2}-\frac{1}{c^{2}} \frac{\partial^{2}}{\partial t^{2}}\right) \mathbf{A}=-\frac{4 \pi \mathbf{J}}{c}+\frac{1}{c} \frac{\partial}{\partial t} \nabla \phi .
$$

Combining Eq. (3) and $\frac{\partial}{\partial t} \nabla \phi=4 \pi \mathbf{J}_{l}$, where $\mathbf{J}_{l}$ reprents the longitudinal current, we obtain the equation for the propagation of a light wave in plasma

$$
\left(\nabla^{2}-\frac{1}{c^{2}} \frac{\partial^{2}}{\partial t^{2}}\right) \mathbf{a}=\frac{\omega_{p}^{2}}{\alpha \gamma c^{2}}\left(1+\delta n_{e}\right) \mathbf{a} .
$$

Ignoring the perturbation term, Eq. (4) yields the dispersion relation for electromagnetic waves in relativistic hot plasma,

$$
\omega_{0}^{2}=k_{0}^{2} c^{2}+\omega_{p}^{\prime 2} / \alpha .
$$

Note that when $T_{e 0}$ is very high, the plasma dispersion is significantly reduced. Making use of the Taylor expansion to the first order $\gamma^{-1}=\gamma_{0}^{-1}-\mathbf{a}_{0} \cdot \widetilde{\mathbf{a}} / \gamma_{0}^{3} \alpha^{2}$, then the first-order perturbation of Eq. (4) is reduced to

$$
\left(\frac{\partial^{2}}{\partial t^{2}}-c^{2} \nabla^{2}+\frac{\omega_{p}^{\prime 2}}{\alpha}\right) \widetilde{\mathbf{a}}=\frac{\omega_{p}^{\prime 2}}{\alpha}\left(\frac{\mathbf{a}_{0}^{2} \cdot \widetilde{\mathbf{a}}}{\gamma_{0}^{2} \alpha^{2}}-\mathbf{a}_{0} \delta n_{e}\right) .
$$

Equations (2) and (6) generally describe the nonlinear coupling between the electromagnetic wave and the plasma oscillations for SRS in hot plasma. In one-dimension case, assuming the perturbation forms as $\delta n_{e}=\left(n_{1} / 2\right) e^{i \psi}+$ c.c.,$\widetilde{\mathbf{a}}=$ $\left(\mathbf{a}_{+} / 2\right) e^{i \psi_{+}}+\left(\mathbf{a}_{-} / 2\right) e^{i \psi_{-}}+$c.c., $\mathbf{a}_{0}=\left(\mathbf{a}_{0} / 2\right) e^{i \psi_{0}}+$ c.c., where $\psi=k x-\omega t, \psi_{0}=k_{0} x-\omega_{0} t, \psi_{ \pm}=\left(k \pm k_{0}\right) x-$ $\left(\omega \pm \omega_{0}\right) t$, and considering circularly polarized laser with $\mathbf{a}_{0}^{2}=\left|\mathbf{a}_{0}\right|^{2} / 2=a_{0}^{2} / 2$ and $\gamma_{0}=\sqrt{1+a_{0}^{2} / 2 \alpha^{2}}$, we obtain the dispersion relation for the SRS instability

$$
\frac{\omega_{p}^{\prime 2} a_{0}^{2}}{4 \alpha^{3} \gamma_{0}^{2}}\left(\frac{c^{2} k^{2} \alpha}{D_{e}}+1\right)\left(\frac{1}{D_{+}}+\frac{1}{D_{-}}\right)=1,
$$

where $D_{e}=-\alpha \omega^{2}+\omega_{p}^{\prime 2}+3 k^{2} \theta_{t e}^{2} / \gamma_{0}, D_{ \pm}=-\omega_{ \pm}^{2}+k_{ \pm}^{2} c^{2}+$ $\omega_{p}^{\prime 2} / \alpha=-\omega^{2}+k^{2} c^{2} \pm 2\left(k_{0} k c^{2}-\omega_{0} \omega\right)$. If $\alpha=1$ and $\theta_{t e}=0$, Eqs. (5) and (7) describe cold plasmas case. ${ }^{7}$

For a tenuous plasma, one can find approximate solution$\mathrm{s}$ of Eq. (7). For forward Raman scattering, we take $\omega=$ $\omega_{p}^{\prime} / \sqrt{\alpha}+i \Gamma_{F R S}, c k=\omega_{p}^{\prime} / \sqrt{\alpha}$, and assume that $\Gamma_{F R S} \ll$ $\omega_{p}^{\prime} / \sqrt{\alpha} \ll \omega_{0}$. Substituting these expressions into Eq. (7), we obtain the maximum growth rate for the forward scattering

$$
\Gamma_{F R S}=\frac{\omega_{p}^{\prime 2} a_{0}}{\sqrt{8} \alpha^{2} \gamma_{0} k_{0} c} .
$$

For backward Raman scattering, we set $D_{-}=0, \omega=$ $\omega_{e k} / \sqrt{\alpha}+i \Gamma_{R B S}$, where $\omega_{e k}=\sqrt{\omega_{p}^{\prime 2}+3 k^{2} \theta_{t e}^{2} / \gamma_{0}}$. Solving for $\mathrm{k}$ from Eq. (7), we get $k=k_{0}+$ $\sqrt{-\omega_{p}^{\prime 2} / \alpha+\left(\omega_{e k 0} / \sqrt{\alpha}-\omega_{0}\right)^{2}} / c$ which corresponds to the maximum growth rate for the backward scattering, where $\omega_{e k 0}=\sqrt{\omega_{p}^{\prime 2}+3 k_{0}^{2} \theta_{t e}^{2} / \gamma_{0}}$. In the limit of $\Gamma_{R B S} \ll \omega_{e k}$, the maximum growth rate turns out to be,

$$
\Gamma_{R B S}=\frac{\omega_{p}^{\prime} a_{0} c k}{4 \alpha \gamma_{0} \sqrt{\omega_{e k}\left(\omega_{0} \sqrt{\alpha}-\omega_{e k}\right)}} .
$$

Equations (8) and (9) show that the thermal temperature suppresses the instability growth rates. If $\alpha=1$ and $\theta_{t e}=0$, the maximum growth rates reduce to the cold plasma limit. ${ }^{7,21}$ For $\alpha=1$ and $\gamma_{0}=1$, they exhibit non-relativistic laser case with the Maxwellian distribution. ${ }^{22}$

To understand the thermal effects on SRS, we have numerically solved Eq. (7) and plotted the real and imaginary parts of $\omega$ in Fig. 1, for temperatures, $T_{e 0}=1 \mathrm{keV}, 100 \mathrm{keV}, 800$ $\mathrm{keV}, 1 \mathrm{MeV}$ and $1.5 \mathrm{MeV}$, and the corresponding $\alpha=1.005$, $1.549,6.530,8.051$ and 11.899 , respectively. In our numerical analyses, all the frequency terms are normalized by $\omega_{0}$, and wave vector $k$ is normalized by $\omega_{0} / c$. The solutions of Eq. (7) define the instability regions, where the linear growth rates exist. Figure 1(a) gives the instability growth rates for the non-relativistic intensity laser case, where the instabilities are found in limited $k$ space. It shows that the maximum growth rates of both forward and backward scattering instabilities are reduced with increase in temperature, while the real part $\operatorname{Re}(\omega)$ corresponding to the maximum growth rate shifts from 0.33 to 0.7 . Also the instability region in $k$-space 

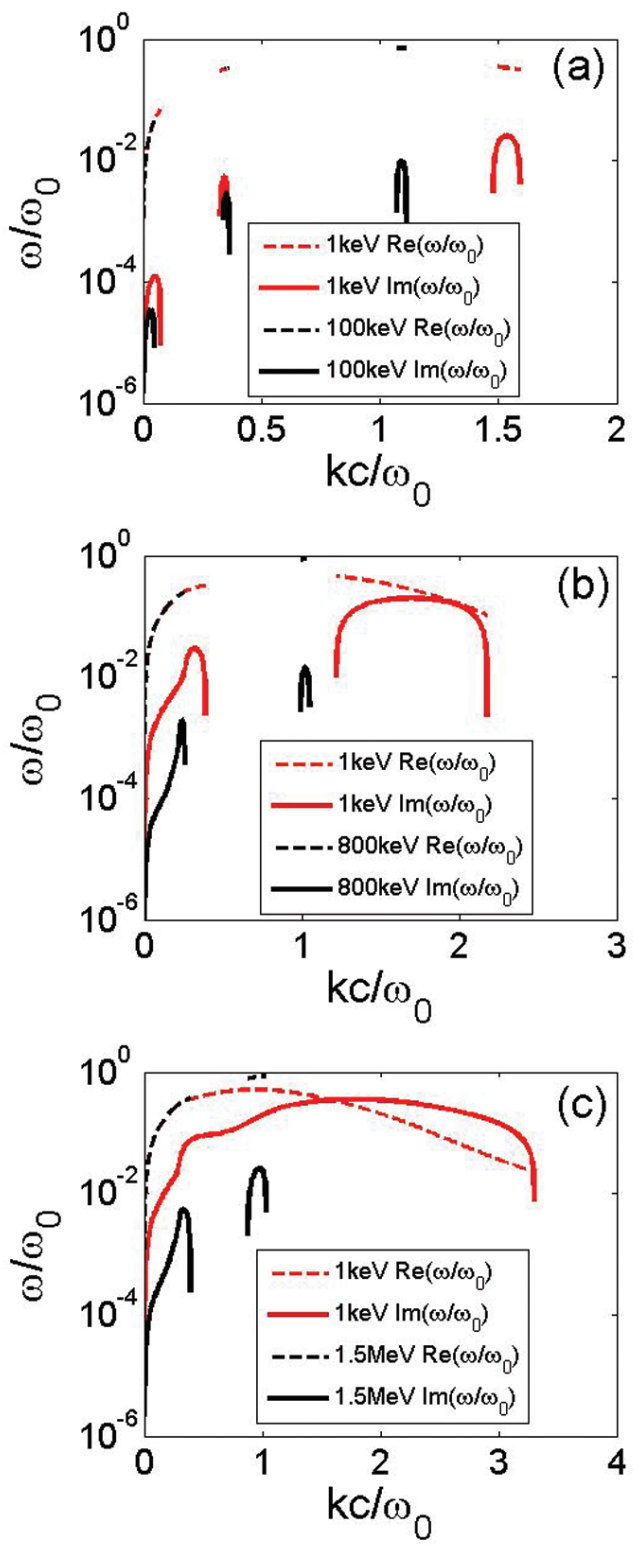

FIG. 1. (color online) Variation of real and imaginary part (growth rate of SRS) of $\omega / \omega_{0}$ with $k c / \omega_{0}$, which are obtained from the numerical solution of Eq. (7). (a) $T_{e 0}=1 \mathrm{keV}$ and $100 \mathrm{keV}$, when $a_{0}=0.1$ and plasma density $n_{e 0}=0.1 n_{c}$. (b) $T_{e 0}=1 \mathrm{keV}$ and 800 $\mathrm{keV}$, when $a_{0}=1$ and plasma density $n_{e 0}=0.1 n_{c}$. (c) $T_{e 0}=1 \mathrm{keV}$ and $1.5 \mathrm{MeV}$, when $a_{0}=3$ and plasma density $n_{e 0}=0.3 n_{c}$

shifts from 1.53 to 1.08 and becomes narrow for the backward scattering as the electron temperature increases from 1 $\mathrm{keV}$ to $100 \mathrm{keV}$. Figures 1(b) and 1(c) show the dispersion curves for the relativistic laser amplitudes. At low temperatures, the instability region becomes wider and growth rates become larger at higher laser amplitude and plasma density, in the meanwhile, the instability can develop even when the plasma density is higher than the quarter critical density as clarified before $^{16,21}$. At $a_{0}=3$ and $n_{e 0}=0.3 n_{c}$, where $n_{c}=m_{e} \omega_{0}^{2} / 4 \pi e^{2}$ is the critical density, the backward and forward scattering regions are found to merge with each other. However, when the electron temperature is increased to the $\mathrm{MeV}$, both growth rates and instability regions are reduced considerably. One may note from Fig. 1, that the frequency of the plasma wave $\omega$ increases when the temperature $T_{e 0}$ is increased, which means the frequency of backscattering light $\omega_{s}=\omega_{0}-\omega$ will decrease. This is clear from the behavior of the real part $\operatorname{Re}(\omega)=\omega_{e k} / \sqrt{\alpha}$ that, when $T_{e 0}$ increases $\operatorname{Re}(\omega)$ grows quickly for smaller values of $k$ and tends to saturate at higher values of $k$.

\section{B. Dispersion relation of SBS}

To derive the dispersion relation for SBS, we consider S$\mathrm{BS}$ as a low frequency perturbation, and use the isothermal approximation in Eq. (1), neglecting the electron inertial ter$\mathrm{m}$. Linearizing the continuity equation for ions and assuming $n_{e 0}=Z n_{i 0}$ and $n_{e 1} \approx Z n_{i 1}$, we obtain the density perturbation equation as follows ${ }^{15}$

$$
\left(\frac{\partial^{2}}{\partial t^{2}}-c_{s}^{2} \nabla^{2}\right) \delta n_{e}=\frac{Z m_{e} c^{2}}{\gamma_{0} m_{i} \alpha} \nabla^{2}\left(\mathbf{a}_{0}^{2} \cdot \widetilde{\mathbf{a}}\right),
$$

where $c_{s}=\sqrt{\left(Z T_{e 0}+3 T_{i 0}\right) / m_{i} c^{2}}, Z$ is the ion charge. The wave equation is the same as Eq. (6). Combining Eqs. (6) and (10), we obtain the dispersion relation for SBS

$$
\frac{\omega_{p}^{\prime 2} a_{0}^{2}}{4 \alpha^{3} \gamma_{0}^{2}}\left(\frac{Z m_{e} c^{2} k^{2} \alpha \gamma_{0}}{D_{i} m_{i}}+1\right)\left(\frac{1}{D_{+}}+\frac{1}{D_{-}}\right)=1
$$

where $D_{i}=k^{2} c_{s}^{2}-\omega^{2}$, and $D_{ \pm}$is defined as before.

We can find approximate solutions for Eq. (11) under certain conditions. For forward Brillouin scattering, we consider high electron temperature case, i.e., $k c_{s} \simeq \omega_{p i}$ or $k \theta_{t e} \simeq \omega_{p}$, where $\omega_{p i}=\omega_{p} \sqrt{Z m_{e} / m_{i}}$. Substituting $\omega=\omega_{p i}+i \Gamma_{F B S}$ into Eq.(11), we obtain the maximum growth rate for the forward scattering

$$
\Gamma_{F B S}=\frac{\omega_{p i} \omega_{p}^{\prime} a_{0}}{\sqrt{8 \alpha^{3}} \gamma_{0} \omega_{0}} .
$$

For backward Brillouin scattering, we have $D_{-}=0$ and $k=$ $2 k_{0}-2 c_{s} \omega_{0} / c^{2}$. Taking $\omega=k c_{s}+i \Gamma_{B B S}$, then the maximum growth rate for the backward scattering turns out to be

$$
\Gamma_{B B S}=\frac{\omega_{p}^{\prime} a_{0} c}{2 \sqrt{2} \alpha} \sqrt{\frac{k_{0} Z m_{e}}{m_{i} c_{s} \omega_{0} \gamma_{0}}} .
$$

For $\alpha=1$ and $\gamma_{0}=1$, the Eqs. (11) - (13) become to nonrelativistic case. $^{22}$

We have solved Eq. (11) numerically to examine the effect$\mathrm{s}$ of relativistic plasma temperature on SBS. As an example, we take $m_{i} / Z m_{e}=1836$. Figure 2(a) shows the variation of growth rate for non-relativistic laser amplitude $a_{0}=0.1$. When the electron temperature increases, both the instability region and maximum growth rate decreases. The real part $\operatorname{Re}(\omega)$ corresponding to the maximum growth rate shifts from 

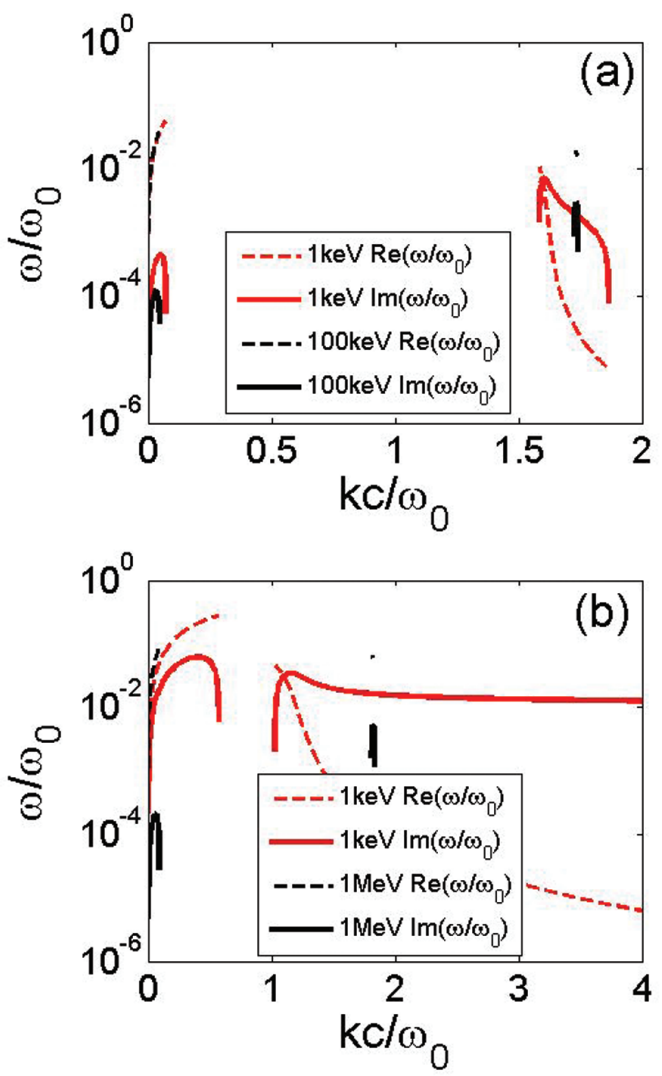

FIG. 2. (color online) Variation of real and imaginary part (growth rate of SBS) of $\omega / \omega_{0}$ with $k c / \omega_{0}$, which are obtained from the numerical solution of Eq. (11). (a) $T_{e 0}=1 \mathrm{keV}$ and $100 \mathrm{keV}$, when $a_{0}=0.1$ and plasma density $n_{e 0}=0.36 n_{c}$. (b) $T_{e 0}=1 \mathrm{keV}$ and 1 $\mathrm{MeV}$, when $a_{0}=1$ and plasma density $n_{e 0}=0.9 n_{c}$.

0.003 to 0.015 . However, the SBS still exists even when $T_{e 0}$ $=100 \mathrm{keV}$. This will be examined in our particle-in-cell simulation in the next section. From Eq. (5) we know that as the temperature increases, $k_{0}$ increases. For backward Brillouin scattering we have $k=2 k_{0}-2 c_{s} \omega_{0} / c^{2} \simeq 2 k_{0}$. Hence $k$ shifts from 1.6 to 1.73 when $T_{e 0}$ increases from $1 \mathrm{keV}$ to 100 $\mathrm{keV}$. Figure 2(b) shows the growth rate for relativistic laser amplitude $a_{0}=1$. When $T_{e 0}=1 \mathrm{keV}$, the instability region is very large, and the maximum growth rate is high. When the temperature $T_{e 0}=1 \mathrm{MeV}$, the instability region becomes very narrow, and the maximum growth rate decreases significantly. Note that when $T_{e 0}=1 \mathrm{MeV}$, the imaginary part of the forward scattering is even larger than the back scattering.

\section{PIC SIMULATIONS}

\section{A. Simulation results of SRS}

To validate the theoretical analysis given in section II, we carried out simulations using the one dimension particle-incell (1D-PIC) code KLAP ${ }^{23}$. In our simulations, the JüttnerSynge distribution is taken when $T_{e 0}>500 \mathrm{keV}$. The length
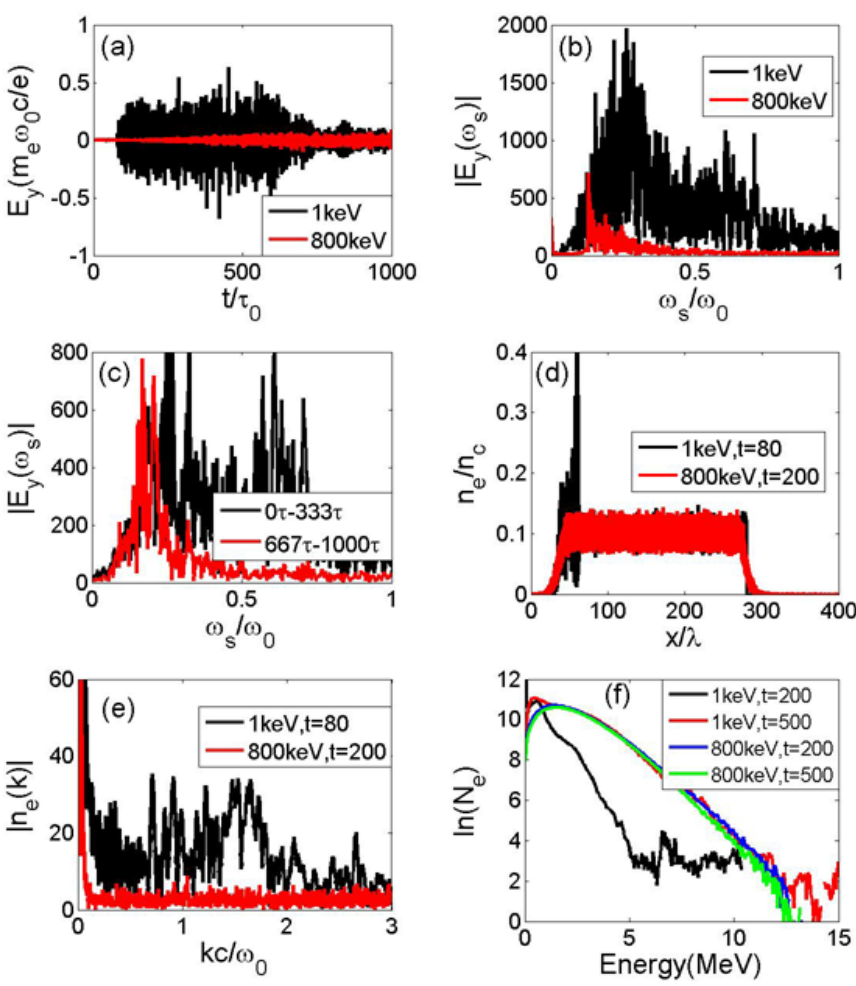

FIG. 3. (color online) PIC simulations of SRS, where the incident laser amplitude and plasma electron density are $a_{0}=1$ and $n_{e 0}=$ $0.1 n_{c}$ and the plasma electron temperature is $T_{e 0}=1 \mathrm{keV}$ or $T_{e 0}=$ $800 \mathrm{keV}$. (a) Temporal profiles of the backscattering light. (b) Fourier transform of the back scattered light. (c) Fourier transform of the back scattered light in different time windows for the case with $T_{e 0}=$ $1 \mathrm{keV}$. (d) Electron density distributions at different time. (e) Spatial Fourier transform of plot (d). (f) Energy distributions of electrons at $200 \tau$ and $500 \tau$ for different initial electron temperatures. $N_{e}$ is the relative electron number.

of our simulation box is $450 \lambda$ with 200 cells per wavelength, where $\lambda$ is the incident laser wavelength. We put 50 particles per cell. Left vacuum and right vacuum regions occupy $30 \lambda$ and $170 \lambda$, respectively, the rest is occupied by plasma. In the front of the plasma, a $10 \lambda$ long linear density ramp is added. The homogeneous plasma density is $n_{e 0}=0.1 n_{c}$. The pump laser is a semi-infinite pulse, which has a $25 \lambda$ ramp before reaching the uniform amplitude at $a_{0}=0.1$ or $a_{0}=1$. In order to emphasis the temperature effect, we take different initial temperatures of plasma. In addition, when laser propagates in plasma, the electrons are heated at the same time.

Figure 3(a) plots the electric field of the backscattered light $E_{y}$, which is normalized by $m_{e} \omega_{0} c / e$, as a function of time under different initial electron temperatures. It shows the amplitude of the backscattering light with initial temperature $T_{e 0}$ $=1 \mathrm{keV}$ increases rapidly, while the amplitude for $T_{e 0}=800$ $\mathrm{keV}$ is comparatively small and grows very slowly with time. This clearly shows that the SRS instability is suppressed significantly at high temperatures. When $t>700 \tau$ where $\tau$ is the wave period of the incident laser, the amplitude of backscattering light at $T_{e 0}=1 \mathrm{keV}$ starts to decrease significantly, then 
it keeps almost a low level comparable to that for $T_{e 0}=800$ $\mathrm{keV}$. This can be considered as a result of plasma heating during the laser propagation when the initial plasma temperature is low. The calculated temperature in the $T_{e 0}=1 \mathrm{keV}$ case is nearly $1 \mathrm{MeV}$ at $700 \tau$, which is high enough to reduce the SRS instability considerably. The temperature suppression effect can also be seen from spectra of the backscattering light given in Fig. 3(b). At $T_{e 0}=1 \mathrm{keV}$, the spectrum of the scattered light is very broad, which is different from the linear dispersion in cold plasma. This kind of behavior is found in nonlinear $\mathrm{SRS}^{24-27}$ and plasma heating. At $T_{e 0}=800 \mathrm{keV}$, only the spectrum with relatively low frequency is dominant. The spectrum peak at 0.13 , i.e., the real part of the plasma frequency is 0.87. It is in agreement with the value of frequency given in Fig. 1(b), i.e., $\operatorname{Re}(\omega) \simeq 0.87$. To show the plasma heating effects on SRS, we calculate the frequency spectrum for the scattered light for the temperature $T_{e 0}=$ $1 \mathrm{keV}$ in two time windows: $0 \tau$ to $333 \tau$ and $667 \tau$ to $1000 \tau$, where the latter time period is when the SRS is considerably reduced. Note that the corresponding spectrum is comparable to the one obtained for $T_{e 0}=800 \mathrm{keV}$. This also suggests that it is the plasma heating that leads to reduced SRS found in Fig. 3(a). Figure 3(d) shows the electron density oscillations associated with the SRS instability. In order to study the linear growth process, we compare electron density for the temperatures $T_{e 0}=1 \mathrm{keV}$ and $800 \mathrm{keV}$ at different time. One may see much stronger fluctuations in plasma for $T_{e 0}=1 \mathrm{keV}$ at $t=80 \tau$ than the one with $T_{e 0}=800 \mathrm{keV}$ at $t=200 \tau$. This means larger instability growth rate occurs at lower temperature $T_{e 0}=1 \mathrm{keV}$. The space Fourier transform of Fig. 3(d) is shown in Fig. 3(e). It displays strong fluctuations in broad $k$ space when $T_{e 0}=1 \mathrm{keV}$, which implies that both SFRS and SRBS have been developed at $t=80 \tau$. In contrast, it is clear from the Fig. 3(e) that there is only a small peak oscillation at $k \simeq 1.04$, for $T_{e 0}=800 \mathrm{keV}$, which is in agreement with Fig. $1(b)$.

The evolution of the plasma electron temperature can directly reflect the SRS development. For $T_{e 0}=800 \mathrm{keV}$, the energy spectrum remains the same in the time interval $t=200 \tau$ to $500 \tau$ as shown in Fig. 3(f). The situation changes dramatically for $T_{e 0}=1 \mathrm{keV}$. In the early stage, due to rapid development of the SRS instability, strong electron plasma wave is produced and electrons are heated quickly. What's more, a high amplitude of backscattering light is produced. It has been found that such high amplitude scattered light can trigger the stochastic heating and acceleration of electrons together with the incident laser. ${ }^{28}$ These lead to rapidly increase in the electron temperature to a level even higher than that found for $T_{e 0}$ $=800 \mathrm{keV}$ case and the formation of a high energy tail of hot electrons. Later at $t=700 \tau$, the SRS is suppressed significantly.

Under non-relativistic conditions our theory still works well, it can be seen from Fig. 4, where the incident laser intensity is $a_{0}=0.1$, and plasma density $n_{e 0}=0.1 n_{c}$. The spectrum of backscattering light obtained at $t=700 \tau$ for $T_{e 0}$ $=1 \mathrm{keV}$ is broad and the amplitude is large as shown in Fig. 4(a). The physical mechanism is similar to the one obtained for $a_{0}=1, n_{e 0}=0.1 n_{c}$ in Fig. 3(b). For $T_{e 0}=100 \mathrm{keV}$,
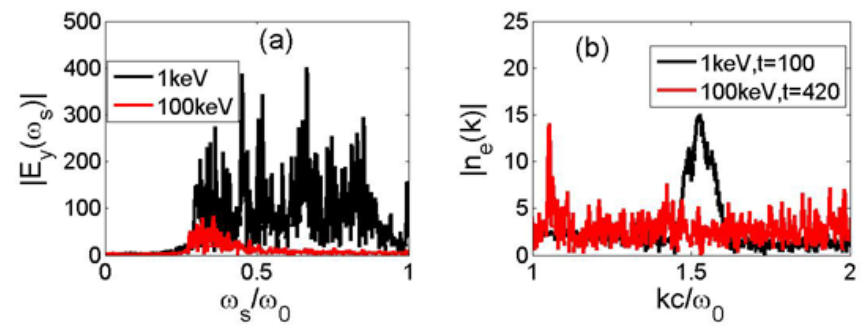

FIG. 4. (color online) PIC simulations of SRS, where the incident laser amplitude and plasma electron density are given by $a_{0}=0.1$ and $n_{e 0}=0.1 n_{c}$ and the plasma electron temperature is $T_{e 0}=1$ $\mathrm{keV}$ or $T_{e 0}=100 \mathrm{keV}$. (a) Fourier transform of the back scattered light. (b) Space Fourier transform of electron density distributions in longitudinal direction.

the scattered light intensity is much smaller than the intensity for $T_{e 0}=1 \mathrm{keV}$. The spectrum of backscattering wave peaks at $\omega_{s} \simeq 0.3$, i.e., $\omega \simeq 0.7$ which is the same as Fig. 1(a). In order to separate nonlinear effects, we compare $k$ modes for the temperatures $T_{e 0}=1 \mathrm{keV}$ and $100 \mathrm{keV}$ at different times, as shown in Fig. 4(b). When $T_{e 0}=1 \mathrm{keV}$, the unstable $k$ modes are broad with a peak at 1.53 , which is in agreement with the results obtained in Fig. 1(a). For $T_{e 0}=100 \mathrm{keV}$, the unstable $k$ modes are narrowed and the peak shifts to 1.05 , which is very close to the value 1.08 obtained from Fig. 1(a). Hence the results obtained using PIC simulations are in close agreement with the theoretical analysis.

\section{B. Simulation results of SBS}

From the analysis given in Sec. IIA, we found that the SRS instability can develop even when the density is higher than the quarter critical density, when the incident laser intensity becomes relativistic, as shown in Fig. 1(c). Therefore, it is preferable to study the effects of plasma temperature on SB$\mathrm{S}$ with non-relativistic laser amplitudes. We take normalized laser amplitude $a_{0}=0.1$ and electron density $n_{e 0}=0.36 n_{c}$, without a density ramp in front of the plasma. For a comparative study we use the electron temperatures $T_{e 0}=1 \mathrm{keV}$ and $100 \mathrm{keV}$, and take $m_{i} / Z m_{e}=1836$. With the given light intensity and simulation time, plasma expansion is not very strong. So the left vacuum occupies $30 \lambda$, and the right vacuum occupies $120 \lambda$. Other parameters are the same as the SRS simulation.

From Fig. 5(a), one can see the amplitude of the backscattered light increases quickly and saturates in a short interval of time at $T_{e 0}=1 \mathrm{keV}$. Meanwhile for $T_{e 0}=100 \mathrm{keV}$, the backscattered light grows very slowly. From Fig. 5(b), one may note that the amplitude of the backscattered light is comparatively larger than the amplitude at $100 \mathrm{keV}$. After linear growth of SBS, nonlinear process develops, during which ions trapped in ion acoustic wave make nonlinear frequency shift. ${ }^{29}$ Thus we see peaks at smaller values of $\omega_{s}$ in Fig. 5(b). The maximum ion acoustic wave frequency obtained is $\omega=0.098$, which is well satisfied with the numerical so- 

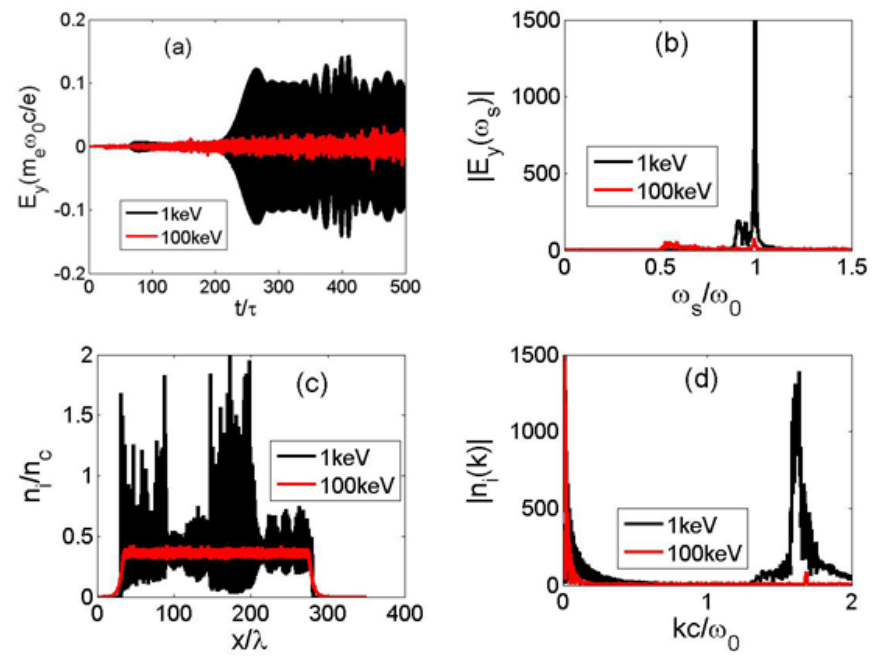

FIG. 5. (color online) PIC simulations of SBS, where the incident laser amplitude and plasma electron density are given by $a_{0}=0.1$ and $n_{e 0}=0.36 n_{c}$ and the plasma electron temperature is $T_{e 0}=1$ $\mathrm{keV}$ or $T_{e 0}=100 \mathrm{keV}$. (a) Backscattered light in time domain. (b) Frequency spectra of the scattered light. (c) is the ion density distributions in longitudinal direction respectively at $500 \tau$. (d) Fourier transform of the ion density distribution in (c).

lution. We now consider the spectrum of the backscattered light at $T_{e 0}=100 \mathrm{keV}$ in Fig. 5(b). Its spectrum has a very small peak at $\omega=0.09$. This means that at $T_{e 0}=100 \mathrm{keV}$ SBS is suppressed significantly. The frequency of backscattering light is approximated to be 0.085 given by our theory. The density profile in Fig. 5(c) shows that at $T_{e 0}=1 \mathrm{keV}$, much stronger ion density fluctuations have been developed. Fourier transform of the ion density distribution shown in Fig. 5(d) indicates that when $T_{e 0}=1 \mathrm{keV}$, the instability occurs at $k \simeq 1.6$. Meanwhile, the wave vector $k$ shifts to 1.69 when $T_{e 0}=100 \mathrm{keV}$. This shift in $k$ agrees well with the expression $k=2 k_{0}-2 c_{s} \omega_{0} / c^{2}$, estimated in Sec. IIB. From the above discussions it is clear that, both the instability region and maximum value are suppressed at high electron temperature as stated in Sec. IIB. This shows that our theory is valid at non-relativistic plasma temperatures.

When laser intensity $a_{0} \gtrsim 0.3$, and plasma density $n_{e 0}>$ $0.25 n_{c}$, kinetic electrostatic electron nonlinear waves ${ }^{30}$ or stimulated electron acoustic wave ${ }^{27}$ will develop. These nonlinear processes will make it hard to observe Brillouin scattering in the backward direction. To study SBS in relativistic intensity region, we set $a_{0}=1, n_{e 0}=0.9 n_{c}$, where strong SBS can be developed at low electron temperature. One has to diagnose the ion density to study SBS. As shown in Fig. $6(\mathrm{a})$, when $T_{e 0}=1 \mathrm{keV}$ the ion density have strong fluctuations in the longitudinal direction, which indicates the onset of intense SBS process. On the contrary, the ion density almost has no fluctuation when $T_{e 0}=1 \mathrm{MeV}$. This means SBS has been reduced under this condition. From Fig. 6(b), it is clear that when $T_{e 0}=1 \mathrm{keV}$ the instability $k$ ranges from 0 to 10 , and stronger fluctuations occur for $k<1$. While at $T_{e 0}$ $=1 \mathrm{MeV}$, the instability strength is very low in comparison
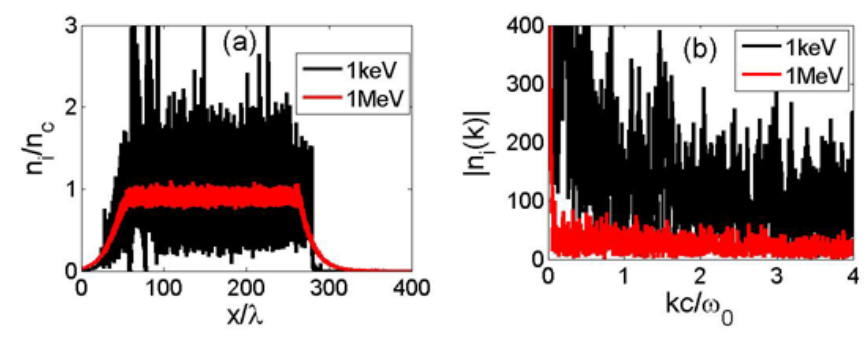

FIG. 6. (color online) PIC simulations of SBS, where the incident laser amplitude and plasma electron density are $a_{0}=1$ and $n_{e 0}=$ $0.9 n_{c}$ and the plasma electron temperature is $T_{e 0}=1 \mathrm{keV}$ or $T_{e 0}$ $=1 \mathrm{MeV}$. (a) is the ion density distributions at $470 \tau$. (b) Fourier transform of the ion density distribution in (a).

to the temperature $T_{e 0}=1 \mathrm{keV}$. These results agree with Fig. 2(b).

\section{SUMMARY}

The effects of relativistic plasma electron temperature on SRS and SBS instabilities have been investigated theoretically and numerically. The dispersion relations for these instabilities are obtained using the relativistic thermal electron fluid model, which uses the kinetic theory and assumes the JüttnerSynge distribution for relativistic hot electrons. The maximum growth rates for these instabilities are obtained, which recover the cold plasma case at temperature close to zero. According to the dispersion relations, it is found that the relativistic electron temperature can reduce the instability region and the growth rates for both SRS and SBS instabilities. Moreover, it decreases the frequency of backscattering light.

To validate the theoretical analysis we have carried out 1DPIC simulations. Both theoretical analysis and PIC simulations showed that the instabilities can be suppressed significantly by hot electron temperatures. When the initial electron temperature is small and the intensity of incident laser pulses is relativistically high, the parametric instabilities can develop rapidly and the plasma electrons can be heated to relativistic high temperatures. Afterwards, the parametric instabilities are significantly suppressed. The PIC simulations are found to be in close agreement with our theoretical analysis.

\section{ACKNOWLEDGMENTS}

This work was supported in part by the National Basic Research Program of China (Grant No. 2013CBA01504), the National Science Foundation of China (Grant Nos. 11121504, 11129503, 11374210 and 11405108), the MOST international collaboration project (Grant No. 2014DFG02330). Yao Zhao appreciates the useful discussions with Magesh Kumar K. K. and Jun Li. M. C. appreciates supports from National 1000 Youth Talent Project of China.

\footnotetext{
${ }^{1}$ T. Tajima and J. Dawson, Phys. Rev. Lett. 43, 267 (1979).
} 
${ }^{2}$ M. Tabak, J. Hammer, M. E. Glinsky, W. L. Kruer, S. C. Wilks, J. Woodworth, E. M. Campbell, M. D. Perry, and R. J. Mason, Phys. Plasmas 1, 1626 (1994).

${ }^{3}$ S. Weng, M. Murakami, H. Azechi, J. Wang, N. Tasoko, M. Chen, Z. Sheng, P. Mulser, W. Yu, and B. Shen, Phys. Plasmas 21, 012705 (2014).

${ }^{4}$ M. Roth, T. Cowan, M. Key, S. Hatchett, C. Brown, W. Fountain, J. Johnson, D. Pennington, R. Snavely, S. Wilks, et al., Phys. Rev. Lett. 86, 436 (2001).

${ }^{5}$ R. Betti, C. Zhou, K. Anderson, L. Perkins, W. Theobald, and A. Solodov, Phys. Rev. Lett. 98, 155001 (2007).

${ }^{6}$ M. Murakami, H. Nagatomo, T. Johzaki, T. Sakaiya, A. Velikovich, M. Karasik, S. Gus' kov, and N. Zmitrenko, Nucl. Fusion 54, 054007 (2014).

${ }^{7} \mathrm{P}$. Gibbon, Short pulse laser interactions with matter (Imperial College Press London, 2005).

${ }^{8}$ S. Wilks, W. Kruer, M. Tabak, and A. Langdon, Phys. Rev. Lett. 69, 1383 (1992).

${ }^{9}$ A. Pukhov and J. Meyer-ter Vehn, Phys. Rev. Lett. 76, 3975 (1996).

${ }^{10}$ P. Shukla and B. Eliasson, Phys. Rev. Lett. 94, 065002 (2005).

${ }^{11}$ G. Li, R. Yan, C. Ren, J. Tonge, and W. Mori, Phys. Plasmas 18, 042703 (2011).

${ }^{12}$ G. Li, R. Yan, C. Ren, T.-L. Wang, J. Tonge, and W. Mori, Phys. Rev. Lett. 100, 125002 (2008).

${ }^{13}$ K.-C. Tzeng and W. Mori, Phys. Rev. Lett. 81, 104 (1998).

${ }^{14}$ J. Bergman and B. Eliasson, Phys. Plasmas (1994-present) 8, 1482 (2001).

${ }^{15}$ G. Li, W. Mori, and C. Ren, Phys. Rev. Lett. 110, 155002 (2013).

${ }^{16}$ S. Guérin, G. Laval, P. Mora, J. Adam, A. Héron, and A. Bendib, Phys.
Plasmas 2, 2807 (1995).

${ }^{17}$ Z.-M. Sheng, K. Mima, Y. Sentoku, and K. Nishihara, Phys. Rev. E 61, 4362 (2000)

${ }^{18}$ T. Grismayer, A. Couairon, P. Mora, J.-C. Adam, and A. Héron, Phys. Plasmas 11, 4814 (2004).

${ }^{19} \mathrm{G}$. Li, Ph. D. thesis (University of Rochester, 2012).

${ }^{20}$ J. G. Siambis, Phys. Fluids 30, 896 (1987).

${ }^{21}$ C. Decker, W. Mori, K.-C. Tzeng, and T. Katsouleas, Phys. Plasmas 3, 2047 (1996).

${ }^{22} \mathrm{~W}$. L. Kruer et al., The physics of laser plasma interactions, Vol. 70 (Addison-Wesley New York, 1988).

${ }^{23}$ M. Chen, Z.-M. Sheng, J. Zheng, Y.-Y. Ma, and J. Zhang, Chin. J. Comput. Phys. 25, 50 (2008).

${ }^{24}$ H. A. Rose and D. A. Russell, Phys. Plasmas 8, 4784 (2001).

${ }^{25}$ W. Rozmus, R. Sharma, J. Samson, and W. Tighe, Phys. Fluids 30, 2181 (1987).

${ }^{26}$ G. Bonnaud, D. Pesme, and R. Pellat, Phys. Fluids B 2, 1618 (1990).

${ }^{27}$ L. Nikolić, M. Škorić, S. Ishiguro, and T. Sato, Phys. Rev. E 66, 036404 (2002).

${ }^{28}$ Z.-M. Sheng, K. Mima, Y. Sentoku, M. Jovanović, T. Taguchi, J. Zhang, and J. Meyer-ter Vehn, Phys. Rev. Lett. 88, 055004 (2002).

${ }^{29}$ E. Williams, B. Cohen, L. Divol, M. Dorr, J. Hittinger, D. Hinkel, A. Langdon, R. Kirkwood, D. Froula, and S. Glenzer, Phys. Plasmas 11, 231 (2004).

${ }^{30}$ A. Ghizzo, T. W. Johnston, T. Réveillé, P. Bertrand, and M. Albrecht-Marc, Phys. Rev. E 74, 046407 (2006). 